\title{
Medien, Kommunikationsformen, kommunikative Gattungen
}

\author{
Christa Dürscheid (Zürich)
}

\begin{abstract}
The aim of this paper is to draw the necessary terminological distinction between media, communication forms and communication genres, and to use this distinction to compare specific communication genres belonging to chat communication. After some preliminary remarks, the following two sections deal with the definition of media and communication forms and the most important characteristics of communication forms, which are illustrated with examples of chat communication. Section 4 introduces the concept of communication genre, and section 5 shows the way communication genres, especially the expert chat and the information chat, can be analyzed within this framework and why it is not possible to do it in the same way with emails and short text messages. In this context the distinction between communication genres and text genres will also be discussed.
\end{abstract}

\section{$1 \quad$ Vorbemerkungen}

Ziel des Beitrags ist, Medien, Kommunikationsformen und kommunikative Gattungen terminologisch voneinander abzugrenzen und auf der Basis dieser Unterscheidung ausgewählte kommunikative Gattungen der Chat-Kommunikation zu vergleichen. Dass zunächst eine terminologische Klärung wichtig ist, zeigt ein Blick in die Literatur. Hier wird z.B. vom Chat als Textsorte (Hess-Lüttich/Wilde 2004), als Diskursart (Storrer 2001), als kommunikative Gattung (Schmidt 2000) oder als Kommunikationsform (Beißwenger 2001) gesprochen; die Abgrenzung bleibt unklar. Auch der Terminus 'Medien' wird nicht einheitlich verwendet. Wie wir im Folgenden sehen werden, steht dahinter eine ganze Reihe von Medienkonzeptionen, und immer noch gilt, was Posner (1986: 297) dazu schreibt: "Der erste Schritt aus dem begrifflichen Chaos muß darin bestehen, die verschiedenen Kriterien auseinander zu halten, die den Verwendungen des Wortes 'Medium' zugrunde liegen."

Der Beitrag gliedert sich wie folgt: In Abschnitt 2 steht die Definition der Termini 'Medien' und 'Kommunikationsformen' im Mittelpunkt. Im Anschluss daran werden die wichtigsten Merkmale zur Unterscheidung einzelner Kommunikationsformen angeführt und an einem Beispiel, der Chat-Kommunikation, illustriert. Abschnitt 4 behandelt das Konzept der kommunikativen Gattungen, das ursprünglich aus der Wissenssoziologie stammt und in der Linguistik eine immer größere Rolle spielt. In Abschnitt 5 wird gezeigt, wie chatspezifische kommunikative Gattungen, nämlich der Beratungs- und der Expertenchat, mit dem Gattungs- 
konzept analysiert werden können und warum es nicht möglich ist, das in analoger Weise in Bezug auf E-Mails und SMS zu tun. In diesem Zusammenhang wird auch auf die Frage der Unterscheidung von kommunikativen Gattungen und Textsorten eingegangen. Abschnitt 6 fasst die wichtigsten Ergebnisse zusammen.

\section{Medien und Kommunikationsformen}

Im öffentlichen Diskurs wird der Terminus 'Medien' seit ca. 15 Jahren geradezu inflationär gebraucht. Man spricht von alten und neuen Medien, von Medienkompetenz und Medienkunde, von Mediothek und Medienlandschaft, von Printmedien, Online-Medien und Multimedia. ${ }^{1}$ Allem zugrunde liegt nicht nur ein Medienkonzept, sondern eine ganze Reihe verschiedener. Dabei wird jeweils eine andere Perspektive als Ausgangspunkt genommen. In der folgenden Übersicht sind in Anlehnung an Posner (1986: 293-297) die wichtigsten Medienkonzepte zusammengestellt (vgl. dazu auch Habscheid 2000). Posner unterscheidet ein biologisches, physikalisches, technologisches, soziologisches, kulturbezogenes und kodebezogenes Medienkonzept. Selbst das ist nur eine Auswahl, ergänzt werden muss die folgende Auflistung z.B. noch um ein kommunikationswissenschaftliches Medienkonzept (vgl. Döring 2003: 37-125).

(1) Medienkonzepte

\begin{tabular}{|c|c|}
\hline Konzept & Beispiel \\
\hline biologisch & audiovisuelle Medien \\
\hline physikalisch & optische/akustische Medien \\
\hline technologisch & Printmedien \\
\hline soziologisch & Verlag/Sendeanstalt \\
\hline kulturbezogen & Roman/Sachbuch \\
\hline kodebezogen & Sprache \\
\hline
\end{tabular}

An dieser Stelle sollen nur die Medienkonzepte knapp erläutert werden, die für die folgenden Ausführungen relevant sind. Das biologische Medienkonzept bezieht Posner (1986: 293) auf die Körperorgane, "die an der Produktion, Distribution und Rezeption von Zeichen beteiligt sind." Beim technologischen Medienkonzept legt er den Schwerpunkt auf die Frage, welche technischen Hilfsmittel für die Produktion von Zeichen benötigt werden und auf welche Weise die Verbindung zwischen dem "Produktionsorgan des Senders und dem Rezeptionsorgan des Empfängers" (Posner 1986: 294) hergestellt wird. In diesem Sinne ist nicht nur die Schreibmaschine ein Medium, sondern auch die Schreibmaschinenseite. Gleiches gilt für Druckmaschine und Drucktext, Fotoapparat und Foto und - ergänzend zu Posners Aufzählung - Computer und Bildschirm. Das kodebezogene Medienkonzept nimmt Bezug auf das jeweils verwendete Zeichensystem. Kodebezogen kann, so Posner (1986: 296), in der Malerei die Gegenüberstellung von gegenständlichen und nicht gegenständlichen Bildern sein, in der Sprache die Entscheidung für eine bestimmte Einzelsprache. Hier ist aus linguistischer Sicht weiter zu fragen, ob das Zeichensystem mündlich oder schriftlich realisiert wird.

\footnotetext{
${ }^{1}$ Das Wort "Multimedia" wurde 1995 von der Gesellschaft für deutsche Sprache gar zum 'Wort des Jahres' gekürt.
} 
Koch/Oesterreicher (1994) sprechen in diesem Zusammenhang von medialer Mündlichkeit bzw. medialer Schriftlichkeit und unterscheiden diese von konzeptioneller Mündlichkeit bzw. Schriftlichkeit. Die Bezeichnungen konzeptionelle Mündlichkeit/Schriftlichkeit stehen außerhalb des hier diskutierten Kontextes, die Bezeichnungen mediale Mündlichkeit resp. Schriftlichkeit dagegen lassen sich unmittelbar auf Posners Medienkonzeptionen beziehen: Die von Koch/Oesterreicher so benannte mediale Schriftlichkeit basiert auf dem technologischen Medienbegriff (= graphisch), die mediale Mündlichkeit auf dem biologischen Medienbegriff (= phonisch) ${ }^{2}$

Im Folgenden lege ich das technologische Medienkonzept zugrunde und schließe mich der Definition an, wie sie von Holly (1997) und Habscheid (2000) gegeben wurde. Medien sind danach "materiale, vom Menschen hergestellte Apparate zur Herstellung/Modifikation, Speicherung, Übertragung oder Verteilung von sprachlichen (und nicht-sprachlichen) Zeichen (Habscheid 2000: 137, vgl. auch Holly 1997: 69 f.). Habscheid und Holly haben mit dieser Definition v.a. die sekundären Medien im Blick. Diese sind dadurch gekennzeichnet, dass auf der Produktions-, nicht aber auf der Rezeptionsseite ein technisches Gerät erforderlich ist. Ein Medium dient aber nicht nur zur Produktion der Zeichen, es kann auch als Hilfsmittel zur Rezeption fungieren. So gilt für die E-Mail-Kommunikation, dass sowohl der Sender als auch der Empfänger ein technisches Gerät (nämlich einen vernetzten Computer) benötigen. Eben das ist ein Kennzeichen von tertiären Medien (vgl. Pross 1972).

Kommen wir nun zur zweiten Frage, zur Unterscheidung von Medien und Kommunikationsformen, genauer: zur Unterscheidung von Kommunikationsmedien und Kommunikationsformen. Ulrich Schmitz (2004: 57) veranschaulicht den Unterschied folgendermaßen: "Medien (z.B. Rundfunk) sind Kommunikationsmittel. Ihre technischen Bedingungen ziehen jeweils bestimmte Kommunikationsformen (z.B. Rundfunksendung) nach sich." Kommunikationsformen sind also kommunikative Konstellationen, die über ein Hilfsmittel erst möglich gemacht werden, aber auch solche, die ohne ein Hilfsmittel auskommen. Letzteres gilt für das Face-to-Face-Gespräch. Aufgrund der Kopräsenz der Teilnehmer ist hier kein Kommunikationsmittel erforderlich. Dagegen ist das zentrale Merkmal der medienvermittelten Kommunikation "die Vergegenwärtigung von Abwesendem" (Saxer 1999: 6). Abwesendes wird über das Medium in die Nähe geholt, Distanzen zwischen Kommunikationspartnern werden durch das Medium überbrückt.

Als Beispiel für die Überwindung von räumlicher Distanz mit Hilfe eines Mediums sei das Telefonieren genannt (synchrone Kommunikation), als Beispiel für die Überwindung von räumlicher und zeitlicher Distanz das Hinterlassen einer Nachricht auf dem Anrufbeantworter (asynchrone Kommunikation). Das Telefon dient mittlerweile aber nicht mehr nur für die fernmündliche, sondern auch für die fernschriftliche Kommunikation. Mindestens drei Formen der Individualkommunikation sind hier möglich: a) Telefonieren, b) 'Simsen', das Verschicken von Textnachrichten (= SMS), und c) 'Mimsen', das Verschicken von Fotos,

\footnotetext{
2 Diesen Hinweis verdanke ich einem anonymen Gutachter.
} 
Audio- und Videodateien in Kombination mit Text (= MMS). ${ }^{3}$ Mehr Kommunikationsformen noch gibt es in dem neuen Medium schlechthin, im vernetzten Computer. Hier laufen alle herkömmlichen Formen der Distanzkommunikation zusammen (z.B. Briefe schreiben, faxen, telefonieren), und neue kommen hinzu (mailen, chatten, bloggen u.a.). Wenn im Folgenden dennoch vor allem die E-Mail- und die Chat-Kommunikation zur Sprache kommen, dann hat das seinen Grund darin, dass diese beiden Kommunikationsformen die Basis jeder computervermittelten, dialogisch ausgerichteten Kommunikation darstellen. Andere, wie die Newsgroup-Kommunikation und die Kommunikation im Instant Messaging, beruhen im Prinzip darauf. Um das deutlich zu machen, seien die beiden Kommunikationsformen kurz vorgestellt:

a) Das Instant Messaging (IM) ist, wie der Chat auch, eine quasi-synchrone Form der Kommunikation. Nicht nur in der Firmen-, gerade auch in der Privatkommunikation unter Jugendlichen wird diese Kommunikationsform immer beliebter und löst häufig schon den EMail-Verkehr ab. Das Programm zeigt an, wer aus dem Kreis der Arbeitskollegen, der Freunde, der Familienmitglieder u.a. gerade online ist, mit wem man also quasi in Echtzeit kommunizieren kann. Die Beiträge folgen unmittelbar aufeinander, bei bestimmten SoftwareProgrammen ist es sogar möglich, dass die Beteiligten zusehen können, wie der andere schreibt, dass die Kommunikation also synchron verläuft. Eine Form des Instant Messaging, die häufig genutzt wird, wird über die Gratissoftware ICQ angeboten (vgl. unter www.icq.de).

b) Newsgroups sind Internet-Diskussionsforen, in denen unterschiedliche Themen, von Problemen mit der Textverarbeitung Word über den Austausch von Kochrezepten bis zum Einsatz neuer Medien behandelt werden (vgl. http://groups.google.de). Wer eine Frage hat, einen Hinweis oder einen Kommentar abgegeben möchte, "postet" seine Mitteilung via EMail in der passenden Newsgroup. In vielen Fällen wird damit ein ganzer Strang von E-Mails (ein "Thread") als Replik auf die eingangs gestellte Frage folgen.

Die Kommunikation in Newsgroups beruht also auf dem Austausch von E-Mails, die Kommunikation im Instant Messaging (IM) auf der Chat-Technologie. Beiden gemeinsam ist, dass es sich um Kommunikationsformen handelt, in denen die Möglichkeit besteht, unmittelbar auf eine vorangehende Sequenz Bezug zu nehmen. Dies freilich geschieht auf unterschiedliche Weise: Im IM kann man direkt antworten, in einer Newsgroup dagegen hat man nur die Möglichkeit, den Teil des Beitrags, auf den man sich bezieht, in den eigenen Text aufzunehmen. Hierzu ein Beispiel aus einer Newsgroup zum Thema Deutsche Sprache. Der Schreiber zitiert aus einer E-Mail, die den Beginn des Threads darstellt:

\footnotetext{
${ }^{3}$ Bekanntlich ist das Simsen (auch "SMS-en") erst vor ca. 10 Jahren zum Telefonat hinzugetreten. 1994 verkaufte Nokia die ersten SMS-fähigen Handys. Das Verschicken von SMS war damals eine kostenfreie, wenig genutzte Zusatzfunktion. Das 'Mimsen' ist dagegen noch jüngeren Datums. Dieser Dienst wird in Deutschland erst seit 2003 angeboten.
} 
(2)

Newsgroup-Beitrag (in de.etc.sprache.deutsch)

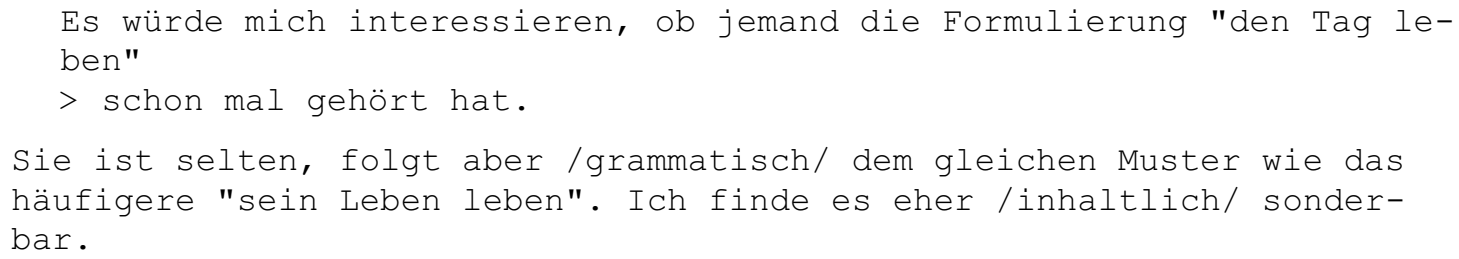

Sequenzen wie in (2) sind charakteristisch für die Struktur von Newsgroup-Artikeln: Im ersten Abschnitt findet sich ein Auszug aus dem Bezugstext, im zweiten Abschnitt folgt die eigene Antwort darauf. Die für E-Mails charakteristische Quote-Technik ermöglicht es hier, einen direkten Bezug zum Inhalt der Frage herzustellen. Solche E-Mails haben, wie im Chat auch, dialogischen Charakter - mit dem einen, wesentlichen Unterschied, dass die Schreiber die Dialoge gewissermaßen im Monolog inszenieren.

\section{Merkmale zur Unterscheidung von Kommunikationsformen}

Ein konstitutives Merkmal von Kommunikationsformen ist das jeweils gewählte Zeichensystem. Sind es Schrift- oder Lautzeichen, handelt es sich also um schriftliche oder mündliche Kommunikation? Weiter sind für die Klassifikation die Kommunikationsrichtung und die Anzahl der Kommunikationspartner relevant. Dabei geht es um die Frage, ob die Kommunikation monologisch oder dialogisch ist bzw. ob es sich um eine Eins-zu-Eins- oder Eins-zuViele-Kommunikation handelt. Letzteres gilt bekanntlich als wesentliches Kennzeichen der massenmedialen Kommunikation über Fernsehen, Radio und Zeitung, aber auch für viele Seiten im WWW. Zwei weitere Merkmale sind die räumliche und zeitliche Dimension der Kommunikation. Beim ersten Punkt ist zu fragen, ob sich die Kommunikationspartner im selben Raum befinden oder eine Distanzkommunikation vorliegt, beim zweiten, ob die Kommunikation synchron oder asynchron verläuft, die Beiträge also unmittelbar aufeinander folgen (wie bei einem Telefongespräch) oder zeitversetzt sind (wie in der Briefkommunikation). Hinzu kommt die grundsätzliche Frage, ob die Kommunikation medial vermittelt ist oder im persönlichen Gespräch stattfindet. Ist Ersteres der Fall, dann stellt sich wiederum die Frage, welchen Einfluss die Nutzung des Kommunikationsmediums auf den Sprachgebrauch hat - und das wiederum ist Gegenstand der Medienlinguistik. ${ }^{4}$

Illustriert sei das Ganze an einem Beispiel, am Chat. Die Kommunikationspartner loggen sich mit einem Spitznamen in einen Chatraum ein (z.B. unter www.chatworld.de). Sie sehen, wie der Dialog auf dem Bildschirm verläuft und können selbst ihre Beiträge über die Tastatur eingeben. Die charakteristischen Merkmale dieser Kommunikationsform sind die folgenden:

\footnotetext{
${ }^{4} \mathrm{Zu}$ diesen und weiteren Merkmalen von Kommunikationsformen vgl. Ziegler (2002). Hier findet sich auch eine ausführliche Diskussion der Unterscheidung von Kommunikationsformen und Textsorten. Danach sind Kommunikationsformen ausschließlich über textexterne Kriterien definiert, Textsorten über textinterne und textexterne Merkmale.
} 
(3) Merkmale der Kommunikationsform Chat

- Zeichentyp: geschriebene Sprache

- Kommunikationsrichtung: dialogisch

- Anzahl der Kommunikationspartner: variabel

- räumliche Dimension: Distanz

- zeitliche Dimension: quasi-synchron

- Kommunikationsmedium: Computer

Im Chat verläuft die Kommunikation über die geschriebene Sprache, sie ist dialogisch, die Anzahl der Kommunikationspartner ist variabel, zwischen ihnen gibt es wie auch beim Telefonieren eine räumliche Distanz. Zu erläutern ist, warum die Kommunikation hier als quasi-synchron bezeichnet wird. Der Grund ist der folgende (vgl. Dürscheid 2003): Die Chatter schicken ihre Beiträge über die Eingabetaste ab, die Mitchatter sehen diese unmittelbar danach auf ihrem Bildschirm und können sofort darauf antworten. Es ist also eine Kommunikation, die quasi in Echtzeit erfolgt. Dennoch ist es nicht berechtigt, den Chat als eine synchrone Form der Kommunikation zu bezeichnen. Die Kommunikationspartner sehen nicht, wie die Äußerung des anderen am Bildschirm entsteht; ${ }^{5}$ sie können also auch nicht intervenieren, unterbrechen, sich simultan äußern - all das, was sie als Zuhörer tun könnten. Insofern ist es falsch, den Chat mit einem Gespräch gleichzusetzen. Es fehlt ein wichtiges Merkmal, die Simultaneität von Produktion und Rezeption der Äußerung. ${ }^{6}$

\section{Kommunikationsformen vs. kommunikative Gattungen}

Thomas Luckmann definiert kommunikative Gattungen als "historisch und kulturell spezifische, gesellschaftlich verfestigte und formalisierte Lösungen kommunikativer Probleme" (Luckmann 1986: 256). So repräsentieren Prüfungs- und Bewerbungsgespräche, Fragen nach dem Weg, Bestellungen im Restaurant bereits verfestigte Lösungen kommunikativer Probleme. Die Beteiligten wissen, wie in diesem Schema zu interagieren haben, welche Aufgaben ihnen zukommen, wie der Handlungsverlauf einzuschätzen ist. Solche Handlungsmuster erleichtern, wie Günthner (1995: 197) schreibt, "die Kommunikation, indem sie die Synchronisation der Interagierenden und die Koordination ihrer Handlungsteile mittels mehr oder weniger vorbestimmter Muster in halbwegs verläßliche, bekannte und gewohnte Bahnen lenken." An anderer Stelle betont Günthner (2000: 19), dass Gattungen als interaktiv erzeugte, dialogische Konstrukte im tatsächlichen Interaktionsprozess beschrieben werden können. Das legt natürlich die Vermutung nahe, dass es primär die mündliche Kommunikation ist, die in der Gattungsanalyse als Untersuchungsgegenstand in Frage kommt. Doch können auch schriftliche Kommunikationsformen damit erfasst werden (s.u.), wenn man den Blick nicht auf einzelne Texte richtet, sondern auf den Interaktionszusammenhang, in dem solche Texte stehen.

\footnotetext{
${ }^{5}$ Wäre dies der Fall, dann läge tatsächlich eine Analogie zum Zuhören beim Sprechen vor.

${ }^{6}$ Zwar gibt es eine Variante der Chat-Kommunikation, bei der die Kommunikationspartner tatsächlich synchron kommunizieren, doch wird diese noch kaum genutzt. Sie können hier sehen, wie der andere schreibt, wie der Text auf dem Bildschirm entsteht. Die Produktion und die Rezeption der Äußerung verlaufen in diesem Fall wie bei einem Gespräch - simultan (vgl. Dürscheid 2003).
} 
Luckmann unterscheidet in seiner Gattungsanalyse weiter zwei strukturelle Ebenen, die Binnenstruktur und die Außenstruktur. Zur Außenstruktur zählt er das soziale Umfeld, in der die Kommunikation stattfindet, die jeweilige Gruppe (z.B. Schüler), die Institution (z.B. Universität), die Geschlechterkonstellation (z.B. Gespräch unter Frauen), also alle die Faktoren, die dem Kontext zuzurechnen sind. Zur Binnenstruktur gehören die verbalen und non-verbalen Bestandteile des kommunikativen Geschehens (z.B. die Wahl bestimmter Ausdrucksmittel, die Prosodie, Gestik und Mimik). Als dritte Ebene kommt eine intersubjektiv-situative Zwischenstruktur hinzu, die von Knoblauch/Günthner (1994) als "situative Realisierungsebene" bzw. von Günthner (2000: 16) als "Interaktionsebene" bezeichnet wird. Auf dieser Ebene geht es um die Abfolge der einzelnen Äußerungen, um die Zuteilung des Rederechts, den Sprecherwechsel, die Themenführung, aber auch um die Frage, in welcher Beziehung die Kommunikationspartner zueinander und zu den in ihrem Gespräch genannten Personen stehen. Die Untersuchungsmethoden der Konversationsanalyse haben hier ihren Anknüpfungspunkt (vgl. Ayaß 2004).

Auf welcher Ebene ist nun das Medium einzuordnen? Im Luckmann'schen Ansatz spielte diese Frage keine Rolle. Das Gattungskonzept war zunächst nur für die Analyse natürlicher Gespräche im Rahmen der Face-to-Face-Kommunikation vorgesehen, die Medienkommunikation blieb ausgeblendet. Anders im linguistisch-anthropologischen Ansatz: Hier findet sich der Hinweis, das Medium gehöre zur binnenstrukturellen Ebene (Günthner 2000: 16; analog dazu auch Günthner 1995: 202). Allerdings möchte ich an dieser Zuordnung Zweifel anmelden: Wenn Günthner (2000: 16) z.B. schreibt, "[a]uch das Medium (mündlich bzw. schriftlich, face-to-face bzw. medial vermittelt) gehört zur binnenstrukturellen Ebene der Gattungsanalyse", dann verbindet sie hier zwei Ebenen, mündlich bzw. schriftlich und face-to-face bzw. medial vermittelt. Was die Unterscheidung von mündlich/schriftlich betrifft, so ist es sicher berechtigt, diese der binnenstrukturellen Ebene zuzuordnen. Ob gesprochen oder geschrieben wird, ist ein internes Merkmal der Kommunikation. Was dagegen die Frage betrifft, ob die Kommunikation medial vermittelt ist oder nicht, so schafft dieses Merkmal erst den Rahmen, in dem sich die Interaktion vollzieht. Es gehört daher zur außenstrukturellen Ebene.

Worin besteht nun aber der Unterschied zwischen kommunikativen Gattungen und Kommunikationsformen? Kommunikationsformen bilden den äußeren Rahmen des kommunikativen Geschehens, kommunikative Gattungen sind die in der Kommunikation konstruierten Handlungsmuster, die den Beteiligten eine Orientierung geben. Illustriert sei dies am Beispiel der Chat-Kommunikation. Wie weiter oben schon gesagt, klassifiziere ich den Chat als Kommunikationsform; im Beitrag von Schmidt (2000) dagegen wird argumentiert, der Chat sei eine kommunikative Gattung. Als Grund wird angegeben, dass das Medium der gesamten Interaktion strikte Vorgaben setze (vgl. Schmidt 2000: 17). Das berechtigt aber nicht dazu, den Chat selbst als kommunikative Gattung anzusehen. Schmidt berücksichtigt nämlich nicht, dass es beim Gattungskonzept gerade darum geht, verschiedene Handlungsmuster voneinan- 
der zu unterscheiden (Bewerbungsgespräche, Verkaufsgespräche, Unterrichtsgespräche etc.). ${ }^{7}$ Solche Handlungsmuster gibt es mittlerweile auch in der Chat-Kommunikation. So verläuft ein nicht-moderierter Freizeitchat, in dem jeder seine Beiträge ohne bestimmte Themenvorgabe eingeben kann, anders als ein Expertenchat, in dem die Struktur in einem Wechsel von Frage und Antwort festgelegt ist. Das wissen die Teilnehmer im Chat, und sie verhalten sich auch entsprechend: Im Expertenchat stellen sie Fragen und akzeptieren es, wenn der Moderator ihre Beiträge kommentiert, bündelt oder zurückstellt. All das muss nicht eigens ausgehandelt werden, das Handlungsmuster ist bereits verfestigt; es wurde aus herkömmlichen Kommunikationspraktiken übertragen. Im Freizeitchat dagegen gibt es keine Themenfixierung, kein festes Ablaufschema, keine vordefinierte Beziehung zwischen den Kommunikationspartnern. Auch das wissen die Chat-Teilnehmer und verstärken diesen informellen Charakter häufig noch durch die sprachliche Gestaltung ihrer Beiträge. Indem sie das tun, gestalten sie den (Interpretations-)Kontext für ihre Handlungen mit. Dabei handelt sich also um ein Kontextualisierungsverfahren (Terminus von John J. Gumperz, vgl. hierzu im Überblick Auer 1999: 164-174).

Im Folgenden werden zwei Chatbeispiele angeführt, um die Unterschiede zwischen kommunikativen Gattungen zu veranschaulichen. Zunächst folgt ein Mitschnitt aus einem Kölner Freizeitchat, die Zeilen wurden durchnummeriert. Die Spitznamen der Chatter sind jeweils vorangestellt. Der Text enthält viele Merkmale informellen Schreibens, Satzabbrüche, Ellipsen, Gesprächspartikeln etc.

(4) Chat-Mitschnitt 1

(1) Pappnase: Kein Kölsch mehr

(2) VOLLblut: dann ist das einfach da hab ich auch schon gewohnt lach

(3) Nick: lol:voll

(4) VOLLblut: ich hab eine Idee

(5) leeloo: ist uns strengstens verboten, leider

(6) Nick: laß hören

(7) Ole29: leeloo, kannst Du Spanisch?

(8) MrBom: Hat einer "DANKE ANKE" gesehen?

(9) monti: nein

(10) Ole29: nee mrb

(11) Pappnase: Keine Kölnerinnen mehr hier ?

(12) leeloo: ein winziges bißchen, habe mal einen kurs gemacht

(13) MrBom: Komisch wackel mit Kopf

Die Beiträge folgen zeitlich zwar unmittelbar aufeinander, allerdings ist die Zuordnung für einen ungeübten Chatter nicht sofort nachvollziehbar, da die Texte vom Chat-Server nach der Reihenfolge ihres Eingangs angezeigt werden, also quasi nach dem Mühlenprinzip (vgl. Beißwenger 2001) auf dem Bildschirm erscheinen: So fragt Ole in (7) Leeloo, kannst du Spanisch?, die Antwort von leeloo kommt aber erst in (12). Ole stellt hier den Namen voran,

7 In einem späteren Beitrag geht Schmidt allerdings anders vor (vgl. Androutsopoulos/Schmidt 2002). Die in diesem Beitrag vorgestellte Analyse zur SMS-Kommunikation ist vergleichbar mit der im Folgenden vorgetragenen zur Chat-Kommunikation. 
macht also explizit, wen er ansprechen möchte. Dieses Verfahren ist bereits konventionalisiert; es wird v.a. dann verwendet, wenn sich die Chatter in einer größeren Runde befinden.

Das zweite Beispiel stammt aus einem moderierten Chat, der im Anschluss an die ZDFFernsehsendung WiSo stattfand. Die Beiträge werden zunächst von einem Moderator geprüft, bevor sie für alle auf dem Bildschirm angezeigt werden. Hier handelt es sich um einen Expertenchat:

\section{(5) Chat-Mitschnitt 2}

(1) dcco: sind die patientenverfügungen auch für den rettungsdienst verbindlich? es kann ja nicht immer sofort überprüft werden ob die verfügung auch wirklich vom patienten stammt und rechtmäßig ist.

(2) WISO-Experte: In der Notsituation in der der Rettungsdiensts tätig ist, wird man der Patientenverfügung nicht unbedingt folgen, weil man zuwenig Anhaltspunkte dazu hat ob der Patient das Verfügte wirklich noch will.

(3) vacat: Wer kann überhaupt eine Patientenverfügung abgeben?

(4) WISO-Experte: Jeder, der einwilligungsfähig ist.

(5) DirkWeber: Kann prinzipiell jeder (auch der Hausarzt, Bruder) als Vertrauenperson eingesetzt werden?

(6) WISO-Experte: Ja.

Der WiSo-Experte gibt Antworten auf Fragen zum Thema Patientenverfügungen; die FrageAntwort-Sequenzen wechseln hin und her, es gibt keinen freien Gesprächsverlauf. Explizite Namensnennungen sind nicht erforderlich, da der Experte nur jeweils einer Person antwortet (dcco, vacat und DirkWeber). Im Beispiel liegen drei Adjazenzpaare vor, drei Frage-AntwortSequenzen. Wie eng diese Sequenzen aneinander geknüpft sind, zeigt die Antwort in (4). Der WiSo-Experte schreibt hier "Jeder, der einwilligungsfähig ist", macht also eine Äußerung, die unmittelbar auf den vorangehenden Satz Bezug nimmt. Aus syntaktischer Sicht ist der Satz unvollständig, es liegt eine Ellipse vor. Das freilich überrascht nicht: Im Chat handelt es sich um eine dialogische Situation, auf die Rede folgt die Gegenrede. Und in einem solchen Dialog wäre es kommunikativ unangemessen, die bereits bekannte Information, das Thema, zu wiederholen, also z.B. zu antworten: Jeder, der einwilligungsfähig ist, kann eine Patientenverfügung abgeben.

Um nun abschließend den Unterschied zwischen kommunikativen Gattungen und Kommunikationsformen noch einmal deutlich zu machen: Kommunikationsformen stellen die Rahmenbedingungen der Interaktion dar. Sie sind durch bestimmte Merkmale gekennzeichnet (Zeichentyp, Kommunikationsrichtung usw.). Ist die Kommunikationsrichtung dialogisch, dann kann weiter gefragt werden, ob die Interaktion einem bereits verfestigten Handlungsmuster folgt. Ist dies der Fall, dann handelt es sich um eine kommunikative Gattung. 


\section{Die Chat-Kommunikation als Gegenstand der kommunikativen Gattungsanalyse}

Schon jetzt sind Chat-Diskussionen nach politischen Sendungen und Talkshows ein festes Angebot von Fernsehanstalten (vgl. www.zdf.de), und auch das über das Fernsehen offerierte Angebot an Expertenchats wird immer größer. In diesem Abschnitt soll gezeigt werden, wie sich die gattungstheoretische Beschreibung dieser neuen kommunikativen Gattungen gestalten könnte. Nehmen wir als Beispiel den Beratungschat:

1) Die sprachlichen Merkmale des Beratungschats lassen sich auf der binnenstrukturellen Ebene beschreiben. Eine solche linguistische Analyse ist Gegenstand bereits vorhandener Arbeiten zur Chat-Kommunikation (vgl. Beißwenger 2001). Wie wir weiter oben gesehen haben, gehören zur binnenstrukturellen Ebene auch die nicht verbalen Ausdrucksmittel, die im Chat aber keine Rolle spielen, da sich dieser schriftlich vollzieht. ${ }^{8}$ Sie werden also, anders als in der kommunikativen Analyse einer Face-to-Face-Beratung, nicht erfasst.

2) Auf der Interaktionsebene muss das System des Redewechsels, die Teilnehmerkonstellation und die Rezipientenreaktionen betrachtet werden (vgl. Günthner 2000: 17). Bei einem Beratungschat, an dem mehrere Personen teilnehmen, ist es wichtig zu untersuchen, wie das Rederecht weitergegeben wird, wie die Teilnehmer den Turnwechsel organisieren, wie sie das Thema fortführen. Auch die Tatsache, dass die Kommunikationspartner den Adressatenbezug nur durch verbale Mittel zum Ausdruck bringen können, nicht aber durch Körperzuwendung, Blickkontakt oder andere Strategien, schafft im Chat eine neue Situation, die es in der Gattungsanalyse zu berücksichtigen gilt.

3) Auf der außerstrukturellen Ebene spielt die Frage eine Rolle, in welchem kommunikativsozialen Milieu der Beratungschat anzusiedeln ist. Ist es ein Chat zwischen Schülern und Lehrern (vgl. www.schulweb.de), ist es eine politische Gruppierung, die den Chat anbietet, ein universitärer Chat, ein Seelsorge-Chat (vgl. www.kirche.de)? Die Tatsache, dass es sich um einen Chat handelt (und nicht etwa um eine E-Mail-Korrespondenz), ist hier ebenfalls relevant, ebenso, dass das Medium der Computer ist (und nicht z.B. das Telefon). Zwar mag man einwenden, dass der Hinweis auf den Chat ja bereits impliziert, um welches Medium es sich handelt, dieses also nicht separat benannt werden muss. Doch gibt es zum einen Chats, die über das Handy stattfinden; zum anderen sollte grundsätzlich getrennt werden zwischen dem Medium, in dem eine Kommunikation stattfindet, und der Kommunikationsform, die über ein Medium möglich ist. Eine Eins-zu-Eins-Beziehung liegt nicht vor.

An dieser Stelle ist noch einmal wichtig zu betonen: Der Chat als Ganzes ist eine Kommunikationsform, keine kommunikative Gattung. Würde man den Chat als eine kommunikative Gattung betrachten, dann könnte man nicht der Tatsache Rechnung tragen, dass es im Chat unterschiedliche, bereits verfestigte Interaktionsmuster gibt. Hinzu käme, dass man dann auch die E-Mail- und die SMS-Kommunikation als kommunikative Gattungen bezeichnen müsste. Wie wir aber weiter oben gesehen haben, umfasst gerade die E-Mail-Kommunikation ein großes Spektrum an Textsorten (Liebesbriefe, Einladungsschreiben, Geschäftsbriefe, Werbe-

\footnotetext{
8 Jedoch kann man darauf hinweisen, dass es Ausdrucksmittel gibt, die Nonverbales kompensieren (z.B. Smileys, Kommentare wie *traurig sei* oder Akronyme wie lol, vgl. Pankow 2003).
} 
briefe etc.), und auch in der SMS-Kommunikation zeichnet sich bereits eine solche Ausdifferenzierung ab. Darauf weisen auch Androutsopoulos/Schmidt (2002) hin, wenn sie in ihrer Untersuchung zur SMS-Kommunikation in einer Kleingruppe schreiben: "Ähnlich wie der Brief ist SMS u.E. keine einheitliche Gattung, sondern eine Kommunikationsstruktur, auf deren Basis einzelne Gattungen wie etwa 'Geschäfts- vs. Privatbrief' in der sozialen Praxis entwickelt werden" (Androutsopoulos/Schmidt 2002: 53). Sie betonen weiter, dass die Gattungen der Kommunikationsform SMS bislang noch nicht verfestigt seien, dass sie sich in statu nascendi befänden. Eben dies gilt auch für die Gattungen der Kommunikationsform Chat. ${ }^{9}$

Allerdings gibt es einen zentralen Unterschied zwischen dem Chat auf der einen und der SMS- und E-Mail-Kommunikation auf der anderen Seite, weshalb das Gattungskonzept nicht gleichermaßen anwendbar ist: Von seiner Struktur her ist der Chat eine Kommunikationsform, die auf der Sequentialität von Äußerungen und auf dem quasi-synchronen Austausch basiert. Die Teilnehmer können den Interaktionsprozess quasi in Echtzeit miteinander aushandeln, auf die Rede folgt die Gegenrede. ${ }^{10}$ Dies ist eine Voraussetzung, um das Gattungskonzept überhaupt sinnvoll anwenden zu können. Anders ist es in der SMSKommunikation. So werden über das Handy häufig Mitteilungen verschickt, auf die keine Antwort erwartet wird und auf die auch keine Antwort kommt. Dazu gehören Bestellungen beim Pizzaservice, aber auch Nachrichten, die über Rennergebnisse, Wetterprognosen, Bundesligaergebnisse etc. informieren (vgl. www.beamgate.de). Diese SMS sind nicht als kommunikative Gattungen zu klassifizieren. Es sind Texte, die sich hinsichtlich ihrer thematischen Funktion unterscheiden, also unterschiedlichen Textsorten zuzuordnen sind (Werbeschreiben, Info-Schreiben etc.). ${ }^{11}$ Ähnliches gilt auch für viele E-Mails (Werbeschreiben, Buchungsbestätigungen, Info-Briefe etc.). Solche Texte lassen sich allenfalls im Sinne von Günthner (1995) als Minimalgattungen auffassen, die Teil eines komplexen Handlungsschemas sind. ${ }^{12}$ Das trifft z.B. dann zu, wenn die E-Mail als Teilsequenz in einem Bewerbungsverfahren oder die SMS als Teilsequenz in einem Dialog unter Freunden betrachtet wird.

\footnotetext{
9 Ayaß (2004: 16) merkt zum Beitrag von Androutsopoulos/Schmidt (2002) an, dass diese "äußere und innere Formen der SMS [...] als mediale Gattungen" untersuchen. Das freilich trifft nicht zu. Androutsopoulos/Schmidt (2002) sprechen an keiner Stelle von medialen Gattungen, sie betonen vielmehr, dass sie mediale Faktoren ganz aus der Analyse ausklammern würden (vgl. Androutsopoulos/Schmidt 2002: 56).

10 Eben dies unterscheidet den Diskurs vom Text (vgl. Dürscheid 2003): Beim Text liegt keine wechselseitige Kommunikation zugrunde, der Diskurs ist gerade durch die Wechselseitigkeit der Kommunikation gekennzeichnet. Die Unterscheidung ist unabhängig davon, ob gesprochen oder geschrieben wird.

11 Die Unterscheidung in Textsorten steht in enger Verbindung zum Gattungskonzept, doch spielt in der Textsortenklassifikation der dialogisch-interaktive Aspekt keine zentrale Rolle. In der anthropologischlinguistischen Gattungsanalyse ist dieser primär. Vgl. Günthner (2000: 21): "Gattungen werden hierbei nicht etwa als homogene, statische Gebilde mit festgelegten formalen Textstrukturen betrachtet, sondern als Orientierungsmuster für die Produktion und Rezeption von Diskursen."

12 Günthner (1995: 199) unterscheidet Minimalgattungen von komplexen Gattungen und merkt an, dass Minimalgattungen integrierte Bestandteile komplexer Gattungen sein können.
} 


\section{$6 \quad$ Zusammenfassung}

Abschließend seien die wichtigsten Merkmale von Medien, Kommunikationsformen und kommunikativen Gattungen zusammenfassend aufgelistet:

a) Medien sind technische Mittel, mit deren Hilfe Distanzkommunikation möglich ist. Sie stellen aber keine notwendige Voraussetzung für Kommunikation dar; das Face-to-FaceGespräch kommt ohne ein Medium aus.

b) Kommunikationsformen lassen sich u.a. danach unterscheiden, ob sie medienvermittelt oder nicht-medienvermittelt sind, dialogisch oder nicht-dialogisch sind. Kommunikationsformen stellen den Rahmen dar, in dem sich kommunikative Handlungsmuster verfestigen können. Diese werden als kommunikative Gattungen bezeichnet.

c) Die Termini 'kommunikative Gattungen' und 'Textsorten' sind nicht gleichzusetzen. Das Konzept der kommunikativen Gattung basiert auf der Annahme, dass eine dialogische Kommunikation vorliegt, das Textsortenkonzept geht für den prototypischen Fall gerade nicht von dieser Annahme aus. Außerdem werden die Klassifikationskriterien unterschiedlich gewichtet: In der Textlinguistik ist die kommunikative Funktion des Textes zentral für die Zuordnung zu einer Gattung (= Textsorte), in der anthropologischlinguistischen Gattungsanalyse ist es das Zusammenspiel von binnen- und außenstrukturellen Elementen und interaktiver Realisierung, das bei der Zuordnung zu einer kommunikativen Gattung zu berücksichtigen ist.

\section{Literaturangaben}

Androutsopoulos, Jannis/Schmidt, Gurly (2002): "SMS-Kommunikation: Ethnografische Gattungsanalyse am Beispiel einer Kleingruppe". Zeitschrift für Angewandte Linguistik 36: 49-80.

Auer, Peter (1999): Sprachliche Interaktion. Eine Einführung anhand von 22 Klassikern. Tübingen.

Ayaß, Ruth (2004): "Konversationsanalytische Medienforschung". Medien \& Kommunikationswissenschaft 52, 1: 5-29.

Beißwenger, Michael (ed.) (2001): Chat-Kommunikation. Sprache, Interaktion, Sozialität \& Identität in synchroner computervermittelter Kommunikation. Perspektiven für ein interdisziplinäres Forschungsfeld. Stuttgart.

Brinker, Klaus (20015): Linguistische Textanalyse. Eine Einführung in Grundbegriffe und Methoden. 5., durchges. u. erg. Aufl. Berlin.

Döring, Nicola $\left(2003^{2}\right)$ : Sozialpsychologie des Internet. Die Bedeutung des Internet für Kommunikationsprozesse, Identitäten, soziale Beziehungen und Gruppen. 2., vollständig überarbeitete und erweiterte Auflage. Göttingen etc.

Dürscheid, Christa (2003): "Medienkommunikation im Kontinuum von Mündlichkeit und Schriftlichkeit. Theoretische und empirische Probleme". Zeitschrift für angewandte Linguistik 38: 37-56.

Günthner, Susanne (1995): "Gattungen in der sozialen Praxis. Die Analyse kommunikativer Gattungen als Textsorten mündlicher Kommunikation". Deutsche Sprache 25/1: 193-218. 
Günthner, Susanne (2000): Vorwurfsaktivitäten in der Alltagsinteraktion. Grammatische, prosodische, rhetorisch-stilistische und interaktive Verfahren bei der Konstitution kommunikativer Muster und Gattungen. Tübingen. (= Reihe Germanistische Linguistik 221).

Günthner, Susanne/Knoblauch, Hubert (1994): "'Forms are the Food of Faith' - Gattungen als Muster kommunikativen Handelns". Kölner Zeitschrift für Soziologie und Sozialpsychologie 46, H. 4: 693-723.

Habscheid, Stephan (2000): "'Medium' in der Pragmatik. Eine kritische Bestandsaufnahme". Deutsche Sprache 28/1: 126-143.

Hess-Lüttich, Ernest W.B./Wilde, Eva (2004): "Der Chat als Textsorte und/oder Dialogsorte". In: Kleinberger Günther, Ulla/Wagner, Franc (eds.): Neue Medien - neue Kompetenzen? Texte produzieren und rezipieren im Zeitalter neuer Medien. Frankfurt a.M.: 49-71.

Holly, Werner (1997): "Zur Rolle von Sprache in Medien. Semiotische und kommunikationsstrukturelle Grundlagen". Muttersprache 1: 64-75.

Koch, Peter/Oesterreicher, Wulf (1994): "Schriftlichkeit und Sprache". In: Günther, Hartmut/Ludwig, Otto (eds.): Schrift und Schriftlichkeit. Ein interdisziplinäres Handbuch internationaler Forschung. An Interdisciplinary Handbook of International Research. 1. Halbband. Berlin/New York: 587-604.

Luckmann, Thomas (1986): "Grundformen der gesellschaftlichen Vermittlung des Wissens: Kommunikative Gattungen". In: Neidhardt, Fritz, et al. (eds.): Kultur und Gesellschaft. Kölner Zeitschrift für Soziologie und Sozialpsychologie. Sonderheft 27. Opladen: 191-211.

Luckmann, Thomas (1995): "Der kommunikative Aufbau der sozialen Welt und die Sozialwissenschaften". Annali di Sociologia 11, I-II: 45-71.

Pankow, Christiane (2003): "Zur Darstellung nonverbalen Verhaltens in deutschen und schwedischen IRC-Chats. Eine Korpusuntersuchung". Linguistik Online 15, 3/03. (http://www.linguistik-online.de/15_03/pankow.html)

Posner, Roland (1986): "Zur Systematik der Beschreibung verbaler und nonverbaler Kommunikation. Semiotik als Propädeutik der Medienanalyse". In: Bosshardt, Hans-Georg (ed.): Perspektiven auf Sprache. Interdisziplinäre Beiträge zum Gedenken an Hans Hörmann. Berlin/New York: 293-297.

Pross, Harry (1972): Medienforschung. Darmstadt.

Saxer, Ulrich (1999): "Der Forschungsgegenstand der Medienwissenschaft". In: Leonhard, Joachim-Felix et al. (eds.) (1999): Medienwissenschaft. Ein Handbuch zur Entwicklung der Medien und Kommunikationsformen. 1. Teilband. Berlin/New York: 1-14.

Schmidt, Gurly (2000): "Chat-Kommunikation im Internet - eine kommunikative Gattung?". In: Thimm, Caja (ed.): Soziales im Netz: Sprache, Beziehungen und Kommunikationskulturen im Internet. Opladen: 109-130.

Schmitz, Ulrich (2004): Sprache in modernen Medien. Einführung in Tatsachen und Theorien, Themen und Thesen. Berlin.

Storrer, Angelika (2001): "Getippte Gespräche oder dialogische Texte? Zur kommunikationstheoretischen Einordnung der Chat-Kommunikation". In: Lehr, Andrea et al. (eds.): Sprache im Alltag. Beiträge zu neuen Perspektiven der Linguistik. Berlin/New York: 439-466. 
Ziegler, Arne (2002): "E-Mail - Textsorte oder Kommunikationsform? Eine textlinguistische Annäherung". In: Ziegler, Arne/Dürscheid, Christa (eds.): Kommunikationsform E-Mail. Tübingen: 9-32. (= Textsorten 7). 